\title{
Update on central nervous system cytopathology. II. Brain smear technique
}

\author{
$\mathrm{J}$ W Ironside
}

\section{Introduction}

Neurosurgery is facilitated by the use of an intraoperative diagnostic neuropathological service, particularly for biopsy specimens of suspected neoplasms. Two main techniques for the intraoperative diagnosis of neurosurgical biopsy specimens are available: cryostat sections and smear or wet film preparations. The use of cryostat sections for rapid diagnosis is popular, particularly with neuropathologists in North America, but in the United Kingdom there has been a long established tradition for using the smear technique as a means of primary investigation. ${ }^{1}$ The relative advantages of each technique have been discussed before. ${ }^{2-4}$ As most intrinsic brain tumours (either primary or secondary) are of soft or gelatinous consistency, smear preparations can readily be made. However, biopsy specimens of firmer consistency-for example, from meningiomas or neurofibromasare often difficult to smear and so may be more easily diagnosed on cryostat sections. The soft consistency and gelatinous matrix of many intracerebral tumours is often accompanied by oedema, which makes cryostat sections from such tissues prone to ice-crystal artefact even when prepared under the most carefully controlled circumstances. For brain biopsy interpretation, smear preparations have an advantage over other rapid cytological techniques for solid tissues-for example, imprint preparations-in that fine cellular detail is combined with architectural features which are of diagnostic value.

The choice of the most suitable investigative technique obviously depends on individual experience and preference, and many neuropathologists prefer, if possible, to use both techniques on biopsy specimens if suffcient tissue is available. ${ }^{3}$ However, there is little doubt that use of the smear technique alone in experienced hands is capable of great diagnostic accuracy, ${ }^{2}$ and it is this method which is considered below. The smear technique has recently enjoyed renewed popularity among neuropathologists as it requires very little tissue to make a satisfactory preparation, making it the method of choice when a rapid diagnosis is requested on a stereotactic biopsy specimen. ${ }^{5}$ The use of minimally invasive techniques in neurosurgery continues to increase. In experienced hands these highly accurate sampling methods allow a tissue diagnosis to be made on lesions previously considered unsuitable for biopsy, particularly deep-seated thalamic or hypothalamic and brain stem lesions. ${ }^{56}$

\section{Technical considerations}

Although the smear technique is ideally suited to specimens from stereotactic biopsy specimens (which typically measure around $8 \times 1 \times 1 \mathrm{~mm}$ ), it is applicable to much larger lesions, from which multiple sites can be sampled rapidly. This is of particular advantage when considering the extremely heterogeneous composition of most malignant gliomas, which are the commonest intracerebral tumours in adults and account for most specimens on which a rapid diagnosis is requested. ${ }^{7}$

The biopsy specimen should be examined as soon as possible after it has been removed from the patient. Small stereotactic biopsy specimens, in particular, will rapidly dry out if left uncovered for even a short period of time, which tends to ruin cytological preservation in smear preparations. All unfixed brain biopsy specimens should be examined and handled in a class I safety cabinet until the smear preparation has been fixed. To make the smear preparation, a fragment of tissue up to $1 \mathrm{~mm}$ in diameter is placed at one end of a pre-labelled glass slide. This can be divided into smaller pieces if required, and these can then be transferred on to other glass slides to be smeared (fig 1A). The tissue samples should be removed from the biopsy specimen with minimal trauma; in particular, it is advisable to avoid crushing the tissue with forceps as this will seriously impair cytological preservation.

Individual tissue samples on the labelled glass slides are gently crushed by a second slide held at right angles (fig 1B). This slide can be gently rotated to spread evenly the specimen across the base of the labelled slide before it is drawn along the length of the labelled slide (fig 1C). It is important to hold the second slide parallel while making the smear preparation to ensure an even distribution of cells across the labelled slide. Smear preparations of normal brain tissue are usually of a uniform smooth appearance on the slide; in reactive states and in neoplasms the tissues tend to smear with an irregular consistency..$^{23}$ The smear preparation is then fixed immediately, without drying, and stained. A variety of fixatives can be used: $95 \%$ ethanol for two minutes is generally satisfactory. A 

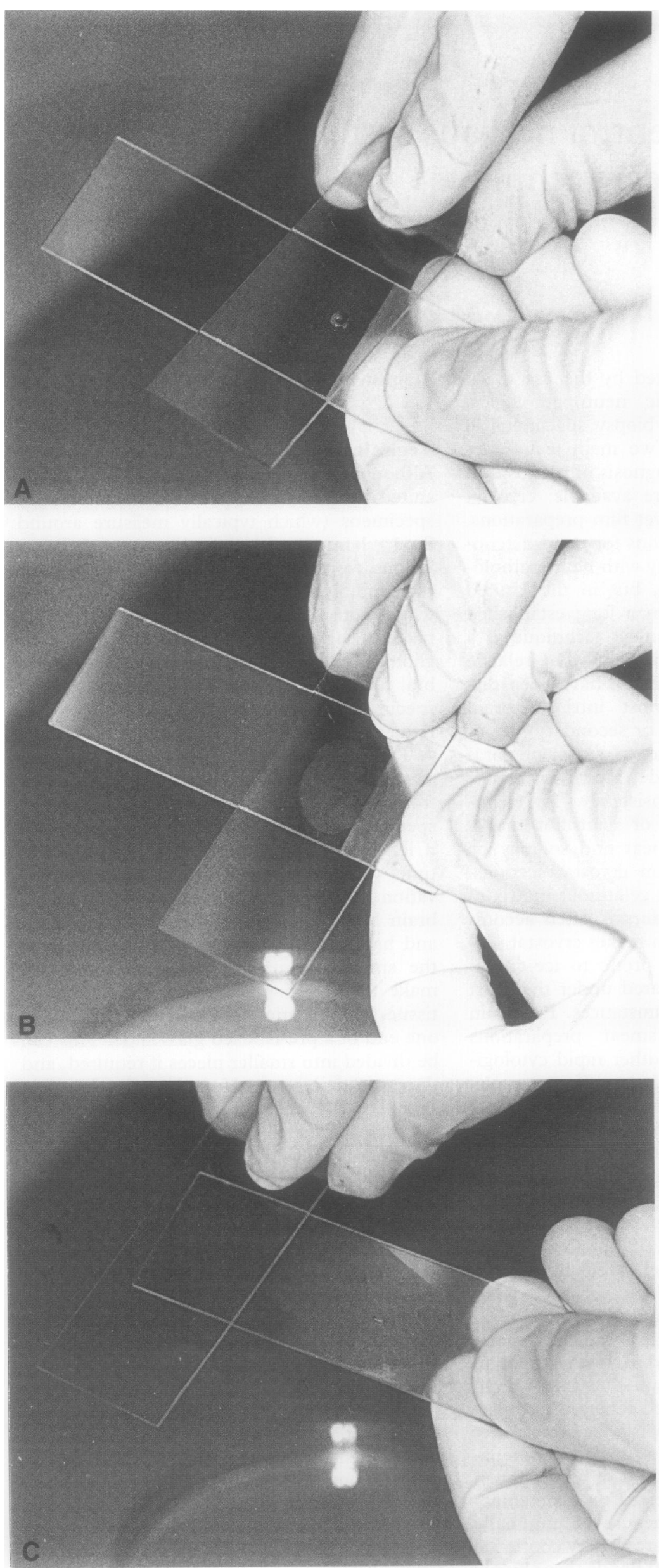

Figure 1 Brain smear technique. (A) The specimen is placed on a slide and a second slide is held at right angles on the specimen. (B) Applying even force with the index finger, the second slide gently crushes the specimen. (C) The second slide is drawn across the first slide with uniform pressure, spreading the specimen evenly. choice of staining techniques is also available: many neuropathologists prefer a toluidine blue stain as this is rapid and gives excellent nuclear detail. ${ }^{2}$ Haematoxylin and eosin is also popular and provides more cytoplasmic detail than other stains, permitting a more direct comparison with cryostat and paraffin wax sections. ${ }^{3}$

In addition to these traditional methods, a microwave oven can be used to shorten the time required for fixation from around two minutes to 30 seconds. $^{8}$ Its advantages with regard to the staining techniques mentioned above are less clearcut, but the microwave oven can be used where other staining techniques, such as immunocytochemistry, are required. Immunocytochemistry using either fluorescence or peroxidase techniques is readily applicable to smear preparations, when cold $\left(4^{\circ} \mathrm{C}\right)$ acetone for two minutes is the fixative of choice. Diagnostic immunocytochemistry is not often used on smear preparations, as in most cases there is enough residual tissue to process into paraffin wax for routine diagnostic techniques. When material for paraffin wax processing is limited, diagnostic immunocytochemistry may be performed if necessary on smears previously stained with haematoxylin and eosin after decolourisation in acid-alcohol. ${ }^{9}$ Some antibodies which are unsuitable for use on paraffin wax embedded material, such as UJ13-A, can be used on smear preparations as well as on cryostat sections after fixation in cold acetone.

\section{Normal cytology}

It is useful to become familiar with the cytology of the normal brain in smear preparations before using this technique for intraoperative diagnosis. This can readily be achieved by sampling various regions of an unfixed necropsy brain with a relatively short post mortem delay, when the various regions of the cerebrum, cerebellum, brain stem and spinal cord can be sampled and stained by a variety of techniques. ${ }^{2}$ Large neurosurgical resection specimens frequently contain portions of cerebral tissue which, although not normal, may be unaffected by tumour and therefore present a useful opportunity for comparison with the lesion under investigation. In smear preparations from the normal brain large neurones can be recognised by their characteristic pyramidal shape, and the presence of a nucleus containing a single prominent nucleolus (fig 2A). Small neurones are less easy to identify with certainty and when numerous - for example, the granular neurones of the cerebellar cortex-can be misinterpreted as neoplastic cells (table). Astrocytes usually have an ovoid, well defined nucleus with stippled chromatin and a characteristic elongated cell body with cytoplasmic processes which are most readily visualised on haematoxylin and eosin stained preparations. Oligodendrocyte nuclei are uniformly round and dark with a more condensed chromatin pattern and scanty cytoplasm (fig 2A). Normal microglial cells are often inconspicuous on smear preparations, 

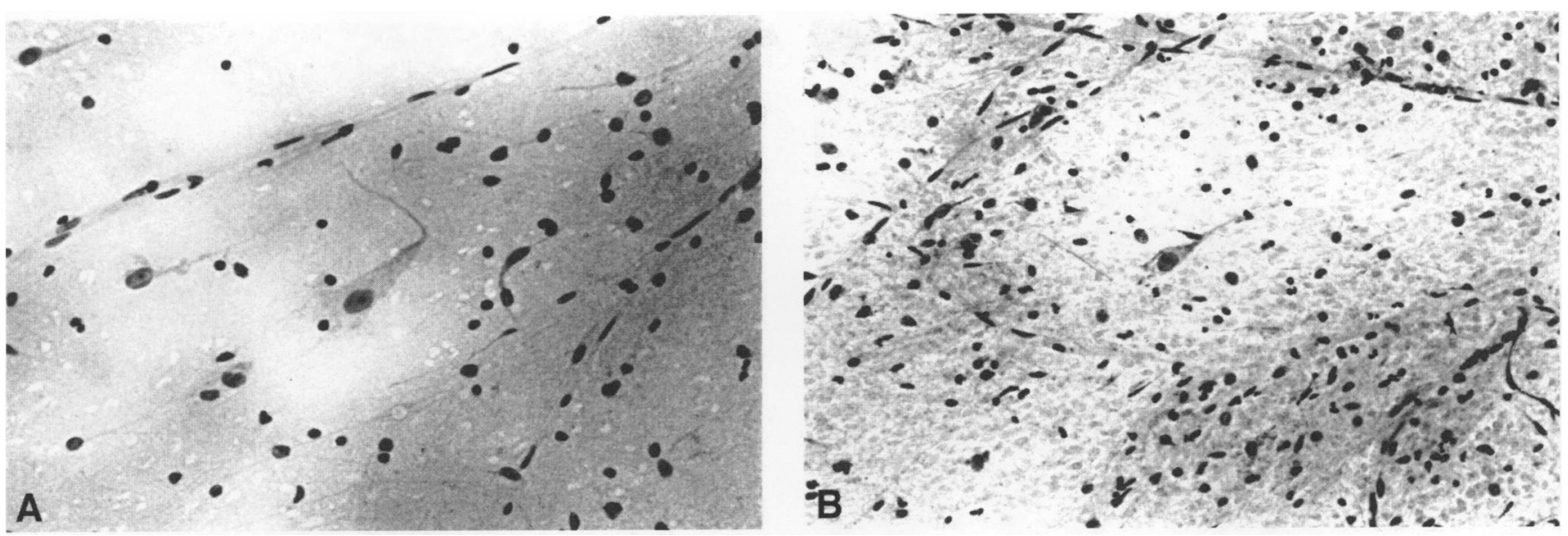

Figure 2 (A) Normal cerebral cortex: a large pyramidal neurone is surrounded by two satellite oligodendrocytes (centre). Ovoid astrocyte nuclei and linear capillary endothelial cell nuclei are also present (haematoxylin and eosin). (B) Reactive gliosis: a pyramidal cortical neurone (centre) is surrounded by increased numbers of endothelial and glial nuclei, including rod-shaped reactive microglial nuclei (haematoxylin and eosin).

Brain smear appearances that may be misdiagnosed as neoplasia

\begin{tabular}{ll}
\hline Tissue type & Misdiagnosis \\
\hline Normal tissue & \\
Choroid plexus & Choroid plexus papilloma \\
Arachnoid granulation & Meningioma \\
Cerebellar granular neurones & Medulloblastoma \\
& Metastatic small cell carcinoma \\
Adenohypophysis & Pituitary adenoma \\
& Metastatic carcinoma \\
Abnormal tissue & Astrocytoma \\
Reactive astrocytosis & Malignant glioma \\
Cerebral infarct with capillary proliferation & Lymphoma \\
\hline Perivascular lymphocytic cuffing &
\end{tabular}

but reactive microglia can be recognised by their rod-shaped nucleus and a characteristic perivascular localisation (fig 2B). Normal capillaries in the brain are thin-walled and delicately branching (fig 2A), although larger arterioles and venules may also be present and can lead the inexperienced observer to suspect capillary endothelial proliferationfrequently present in gliomas. Other normal cellular constituents, such as ependymal and choroid plexus cells, and arachnoidal cells are occasionally sampled in smear preparations. These may be mistaken for neoplastic cells by an inexperienced observer and so caution is needed in their interpretation (table). The smear technique can also be used in the diagnosis of intrinsic spinal cord tumours, intradural and extradural spinal lesions, pituitary and orbital lesions. ${ }^{23}$ Interpretation of biopsy specimens from these sites obviously requires a corresponding degree of familiarity with normal cytology to avoid diagnostic confusion. This is particularly true of tumours affecting the pituitary gland region (table).

\section{Reactive changes}

Most intracranial space-occupying lesions elicit a range of reactive changes in the adjacent brain, including oedema, capillary proliferation, astrocytic and microglial proliferation and inflammation. ${ }^{7}$ These changes can be identified readily in smear preparations (fig 2B), but should not be misinterpreted as neoplasia (table). ${ }^{23}$ This problem may be compounded by the presence of mitotic activity in florid reactive astrocytosis and nuclear pleomorphism in longstanding reactive states. However, most cases of reactive astrocytosis can be identified from the infiltrating edge of an astrocytoma by the relative uniformity of the astrocytic nuclei, the tendency of reactive astrocytes to occur in small clusters which are
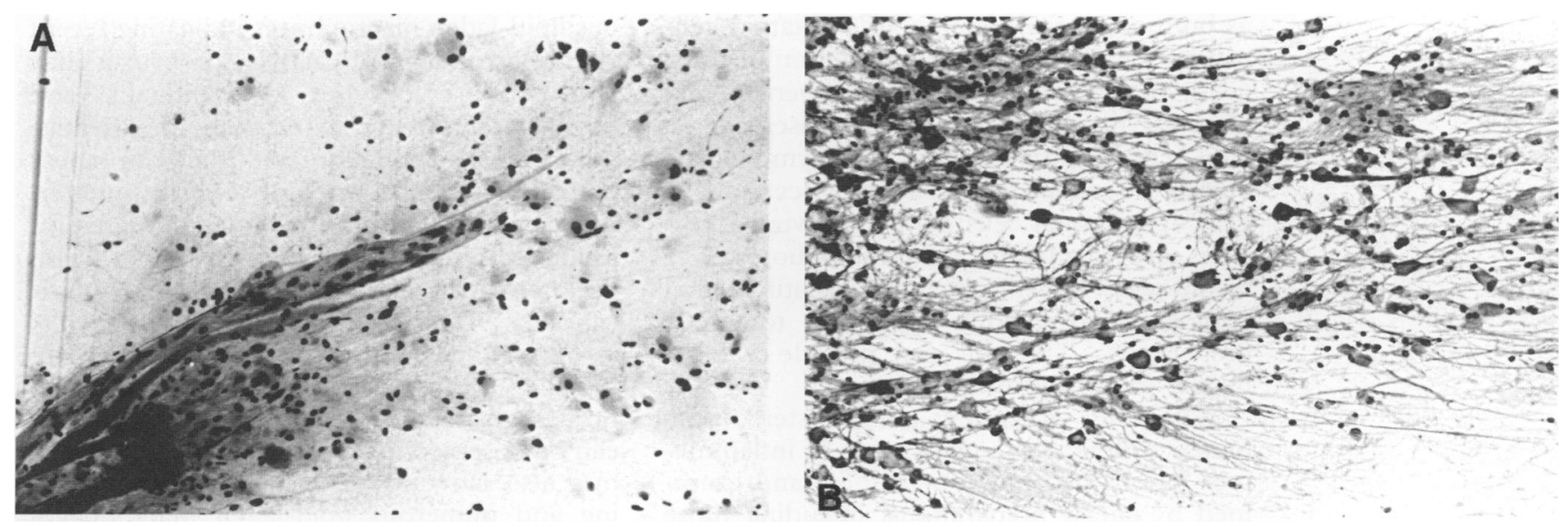

Figure 3 Anaplastic astrocytoma. (A) Hyperplastic capillary endothelium and pleomorphic tumour cell nuclei (including multinucleate cells) are present (toluidine blue). (B) Immunocytochemistry for glial fibrillary acidic protein (same case as in fig $3 A$ ) shows abundant tumour cell cytoplasm and elongated cell processes (haematoxylin counterstain). 

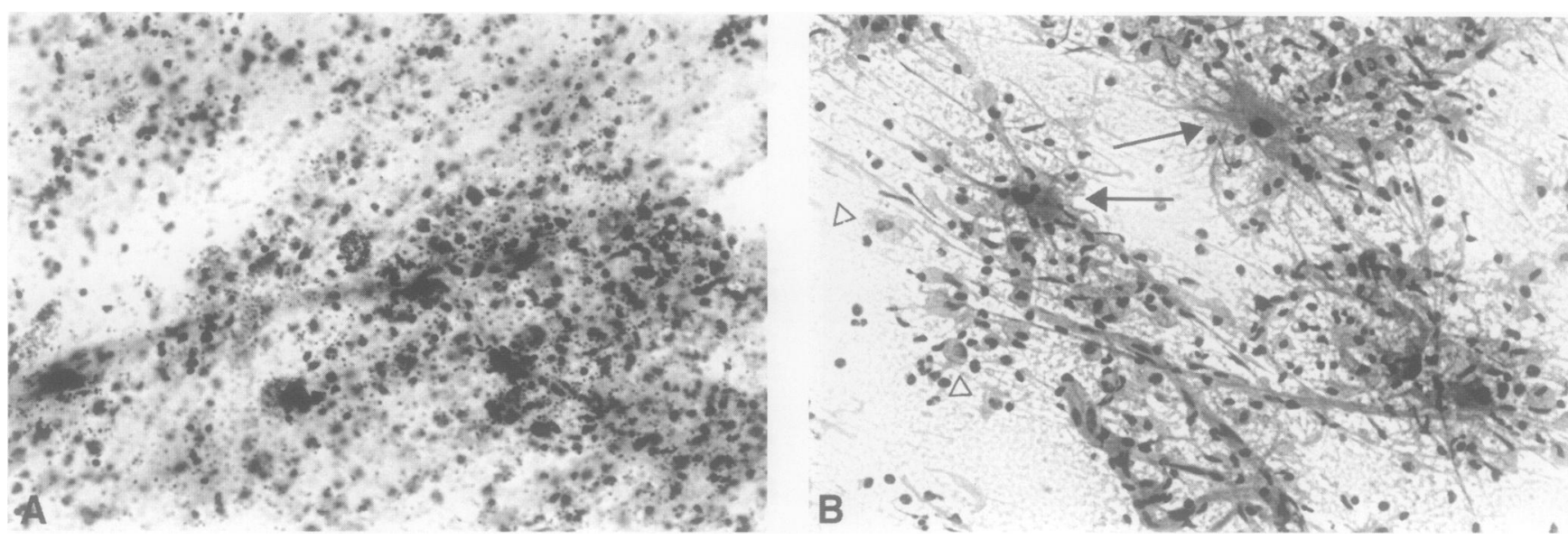

Figure 4 Infections in immunocompromised patients. (A) Toxoplasmosis: in this necrotic tissue a cyst is present (centre) surrounded by numerous small tachyzoites, some of which are within macrophage cytoplasm (haematoxylin and eosin). (B) Progressive multifocal leucoencephalopathy: bizzare enlarged astrocytes (arrows) are present adjacent to capillaries surrounded by lipid-laden macrophages (arrowheads), indicating myelin breakdown (haematoxylin and eosin).

often evenly dispersed throughout the thin part of the smear preparation, and the relation of these cells to capillaries. Capillary endothelial proliferation is more frequently observed in astrocytomas, and the neoplastic astrocytes even at the margins of an infiltrating tumour are frequently aligned around these abnormal blood vessels (fig 3).

Capillary proliferation may also be observed in the tissue around metastatic tumours, in cerebral infarcts (where the vessels are usually thin-walled and surrounded by reactive lipid-laden macrophages), and in inflammatory disorders, including viral encephalitis and cerebral abscesses. ${ }^{2}$ The associated inflammatory changes in the latter two conditions usually permit clear distinction from a neoplasm. It should be remembered that tissue infiltration by neutrophil polymorphs occurs in the early stages of infarction whether this takes place in otherwise normal brain or in a neoplasm, particularly a glioblastoma multiforme. ${ }^{3}$

\section{Inflammatory reactions}

The smear technique is a good way of rapidly studying inflammatory processes in the brain, as both acute and chronic inflammatory cells can be identified and the association of these cells with blood vessels (as in perivascular lymphocytic cuffing) is easily observed. In viral encephalitis perivascular lymphocytic cuffing is readily apparent and occasionally glial or neuronal cells containing viral inclusions are identifiable. ${ }^{2}$ Immunofluorescence techniques specifically to identify viral pathogens are readily applicable to smear preparations, and this can provide a rapid means of diagnostic confirmation.

With the increasing use of stereotactic biopsy techniques, a wide range of inflammatory lesions can now be sampled and examined by smear preparations, including those occuring in patients with HIV related disorders and other immunocompromised persons. Published safety guidelines describe the special precautions required for handling unfixed tissues from HIV seropositive patients ${ }^{10}$; unless there is ready access to a "high-risk" laboratory, it is recommended not to perform cryostat sections or smear preparations on biopsy specimens from patients infected with category 3 pathogens. Given these restraints, it is still possible to achieve an accurate diagnosis using the smear technique if the appropriate laboratory facilities are available. Cerebral toxoplasmosis (fig 4 A) and primary brain lymphoma are among the commoner space-occupying lesions occurring in AIDS, and may be diagnosed in smear preparations. Brain biopsy may be indicated for such a lesion if the patient has failed to respond to anti-toxoplasma treatment; toxoplasmosis can be confirmed on smear preparations using rapid immunofluorescence techniques.

Demyelinating disorders can also be studied using the smear technique. Multiple sclerosis occasionally presents with the clinical features of a space-occupying mass, which usually represents the acute phase of a demyelinating plaque. ${ }^{11}$ Biopsy at this stage in the evolution of the plaque usually shows perivascular lymphocytic cuffing with numerous lipid-laden macrophages indicating recent myelin breakdown. Reactive astrocytosis may also be observed, but it is difficult (and unwise) to attempt to estimate the relative numbers of oligodendrocyte nuclei in smear preparations of cerebral white matter. Another demyelinating disorder-progressive multifocal leucoencephalopathy-may also occasionally be investigated using neurosurgical biopsy. This papovavirus infection results in multiple areas of demyelination which are initially perivascular, accompanied by a bizarre reactive astrocytosis. ${ }^{12}$ These features can be observed in smear preparations, which may also show perivascular lymphocytic cuffing and numerous lipid-laden macrophages (fig 4B). Bizarre reactive astrocytes are usually present, and should not be mistaken for neoplastic cells. 


\section{Neoplastic disorders}

As indicated above, the smear technique is suitable for a wide range of neoplasms involving the central nervous system, including intrinsic and extrinsic primary neoplasms, metastatic lesions, and neoplasms arising in adjacent structures including the orbit and pituitary gland. It is beyond the scope of this article to provide comprehensive guidance on the cytological appearances of the wide range of tumours that may be examined in this way, and the reader is referred to standard texts. ${ }^{23}$ However, there are a number of well recognised difficulties in this field which often relate to problems in tissue sampling, both by the neurosurgeon and the pathologist. The increasing accuracy of stereotactic biopsy techniques now provides a means of obtaining tissue samples from well delineated sites within an intracranial neoplasm, which should usually include tissues from the centre and edge of the lesion. ${ }^{5}$ Comparison of the cytological appearances in these specimens within individual cases is often instructive. The heterogeneous nature of many intrinsic glial neoplasms is well recognised and it is therefore advisable, if at all possible, to sample multiple sites from a biopsy or resection specimen to obtain representative smear preparations of the neoplasm. Should this not be possible, it is important for the neurosurgeon and pathologist to realise that the cytological appearances in a small preliminary biopsy specimen may not be representative of a larger lesion. Close liaison between clinician and pathologist is obviously helpful in this respect, and the pathologist must ensure that adequate clinical and radiological information is available when interpreting small biopsy specimens from a large lesion. ${ }^{3}$ In some circumstances it may be advisable for the pathologist, radiologist, and surgeon to discuss a strategy for biopsy before surgery to ensure that adequate representative tissue samples are obtained.

\section{Recent developments}

DELINEATION OF TUMOUR MARGINS

Even the most sophisticated neuroradiological imaging techniques will not always demonstrate the edges of the infiltrating margin of an intrinsic brain or spinal cord tumour. The accurate delineation of tumour margins is an important consideration when radical neurosurgery or radiotherapy is considered. This can now be studied in serial stereotactic biopsy specimens using the smear technique to give the surgeon rapid information during an operative procedure. This technique has been used to study and classify the patterns of growth in primary intracranial tumours. ${ }^{13}$ As yet, the biological and clinical value of these findings has not been fully established; prospective studies of surgical and irradiation treatments based on this investigation are in progress.

TUMOUR CELL BIOLOGY

Most smear preparations contain areas where tumour cells are spread in a monolayer that is suitable for investigative cytological studies. Investigations of cell proliferation and ploidy studies have been performed using immunocytochemistry and the Feulgen staining technique for DNA. ${ }^{14}$ Antibodies such as Ki-67 and MIB-1 work well on acetone-fixed smear preparations and can be used in quantitative studies of cell proliferation in brain tumours. The Feulgen technique has been used to measure nuclear area, the cellular proliferation index, and ploidy on smear preparations from stereotactic brain tumour biopsy specimens, demonstrating the pronounced cellular heterogeneity within supratentorial gliomas in adults. ${ }^{14}$ Additional work in progress suggests that some of this information is of prognostic importance when considered in association with the patient's age and the histological grade of the tumour.

Smear preparations are also suitable for analysis by in situ hybridisation. Monolayer preparations of tumour cells are particularly suitable for interphase cytogenetic studies using fluorescence in situ hybridisation. Tumours of the astroglial series (astrocytoma, anaplastic astrocytoma, and glioblastoma multiforme) frequently exhibit chromosomal abnormalities, including losses of part or all of chromosomes 10 and 13 and polysomy of chromosome 7. These abnormalities often occur in association with other molecular genetic changes, such as amplification of the EGFR gene, and there is evidence to suggest that the incidence of these abnormalities increases with tumour grade. Fluorescence in situ hybridisation has been used to study chromosomal abnormalities in brain smear preparations, which have the additional advantages of avoiding prolonged culture techniques which might otherwise be necessary to obtain sufficient numbers of cells for analysis, thereby avoiding in vitro artefacts. ${ }^{15}$

\section{Conclusion}

The smear or wet film technique has long been established for the diagnosis of tumours of the brain and associated tissues. The increasing use of minimally invasive neurosurgical techniques has renewed interest in this method, which is of particular value in providing a rapid intraoperative diagnosis on small stereotaxy specimens. The use of the smear technique to examine multiple samples of tissue from intrinsic neoplasms and to investigate infiltrating tumour margins has provided new information on the growth patterns of intrinsic brain tumours which may be of therapeutic potential. The smear technique can also be applied to non-neoplastic lesions, which form an increasing part of the diagnostic workload in many neuropathology laboratories. The fine cytological detail in monolayer smear preparations renders this material particularly suitable for studies of brain tumour biology, including cell proliferation, cell ploidy, and in situ hybridisation studies which are otherwise possible only after prolonged tumour cell culture. 
These and other techniques will also increasingly be applied to brain tumour smear preparations.

I am grateful to $\mathrm{Mr}$ W $\mathrm{M}$ Shade and the staff of the Neuropathology Laboratory, Edinburgh, for skilled technical assistance, to Dr JE Bell for helpful discussion, and to Miss A Boyd for assistance in preparing the manuscript.

1 Russell DS, Krayenbuhl H, Cairns $\mathrm{H}$. The wet film technique in the histological diagnosis of intracranial
tumours; a rapid method. $f$ Pathol Bacteriol 1937;45: tumours:

2 Adams JH, Graham DI, Doyle D. Brain biopsy. The smear technique for neurosurgical biopsies. London: Chapman and Hall, 1981.

3 Franks AJ. Diagnostic manual of tumours of the central ner vous system. Edinburgh: Churchill Livingstone, 1988. 4 Jane JA, Bertrand G. A cytological method for the diagno-
sis of tumors affecting the central nervous system. 7 Neuropathol Exp Neurol 1962;21:400-9.

5 Kitchen N, Bradford R, Thomas DGT. Stereotactic surgery: methods of CT and MRI directed biopsy. In: Thomas DGT, ed. Sterotactic and image directed surgery of brain tumours. Edinburgh: Churchill Livingstone, of brain tumo

6 Thomas DGT, Kitchen ND. Minimally invasive surgery. Neurosurgery. $B M f$ 1994;308:126-8
7 Russell DS, Rubinstein LJ. Pathology of tumours of the nervous system. 5th edn. London: Edward Arnold, 1989. 8 Ainley CD, Ironside JW. Microwave technology in diag nostic neuropathology. $\mathcal{F}$ Neurosci Methods (in press).

9 Laing RW, Smith CML. A useful technique for improving the diagnostic yield from single stereotactic biopsies of brain. Neuropathol Appl Neurobiol 1990;16:529.

10 Advisory Committee on Dangerous Pathogens. HIV-the causative agent of AIDS and related conditions. Second revision of guidelines. London: HMSO, 1990

11 Hunter SB, Ballinger WE, Rubin JJ. Multiple sclerosis mimicking primary brain tumor. Arch Pathol Lab Med 1987;111:464-8.

12 Burger PC, Scheithauer BW, Vogel FS. Surgical pathology of the nervous system and its coverings. 2nd edn. New of the nervous system and its coverings. 2 .
York: Churchill Livingstone, 1991:155-7.

13 Daumas-Duport C, Monsaigneon V, Blond S, Munari C, Musolino A, Chodkiewicz JP, et al. Serial stereotactic biopsies and CT scan in gliomas: correlative study in 100 astrocytomas, oligo-astrocytomas and oligodendrocytomas. F Neuro-Oncol 1987;4:317-28.

14 Salmon I, Levivier M, Cambry I, Rombaut K, Gras T, Pasteels J-L, et al. Assessment of nuclear size, nuclear DNA content and proliferation index in stereotaxic biopsies from brain tumours. $f$ Neuropathol Appl Neurobiol 1993;19:507-18.

15 Low M, Feiden W, Moringlande JR, Zang KD, Henn W. Detection of numerical chromosome abnormalities in brain tumours by fluorescence in situ hybridisation on smear preparations of small tumour biopsies. Neuropathol Appl Neurobiol (in press). 